\title{
AN APPLICATION OF FRACTIONAL INTEGRATION TO CHAIN TRANSFORM THEORY
}

\section{CHARLES FOX}

1. Introduction. In two previous papers, Fox $[2 ; 3]$, I have defined chain transforms and obtained the conditions that a set of functions should form the kernels of a chain transform. In this paper I shall show that the property of forming a set of kernels of a chain transform is largely invariant under the operation of fractional integration. Thus from one set of chain transform kernels we can, by means of this operation, obtain an infinite number of other such sets of kernels.

We shall use here the definition of fractional integration which has been developed largely by Erdelyi [1] and Kober [4].

2. Chain transforms. A chain transform of order $n$ is defined, [3], as a set of $n$ equations divided into two subsets, one of $i$ and the other of $j$ equations, where $i+j=n, i, j$ and $n$ being all positive integers. These subsets of equations are as follows:

$$
\begin{aligned}
g_{p+1}(x) & =\int_{0}^{\infty} r_{p}\left(\frac{x}{u}\right) g_{p}(u) \frac{d u}{u}, \\
\int_{0}^{\infty} l_{q}\left(\frac{x}{u}\right) g_{q+1}(u) \frac{d u}{u} & =g_{q}(x),
\end{aligned}
$$

where $p$ runs through $i$ integers and $q$ through $j$ integers, but not necessarily in consecutive fashion. Between them $p$ and $q$ run through the first $n$ positive integers. The functions $g_{i}(x)$ commence with $g_{1}(x)$ and terminate with $g_{n+1}(x)$.

For the system (1), (2) to be a chain transform of order $n$ we must have (i) $g_{n+1}(x)=g_{1}(x)$, so that there are only $n$ of these functions and (ii) each of the functions $g_{i}(x), i=1,2,3, \cdots, n$, occurs twice in the system (1), (2), once on the right hand side and once on the left. In one of these occurrences it will appear as $g_{i}(x)$ and in the other as $g_{i}(u)$. The functions $r_{p}(x)$ and $l_{q}(x)$ are known as the kernels of the chain transform. When $n=2$ the system reduces to the Generalized Fourier Transform, Titchmarsh [5].

In my two previous papers, $[2 ; 3]$, I have given examples of chain transforms of various orders. An example of order three is as follows:

Presented to the Society April 26,1958, under the title Fractional integration and chain transforms; received by the editors April 9, 1958. 


$$
\begin{aligned}
& g_{2}(x)=\int_{0}^{\infty}\left(\frac{u}{x} e^{-u / x}\right) g_{1}(u) \frac{d u}{u}, \\
& g_{3}(x)=\int_{0}^{\infty} e^{-x / u} g_{2}(u) \frac{d u}{u}, \\
& \int_{0}^{\infty} \frac{1}{\{1+(x / u)\}} g_{1}(u) \frac{d u}{u}=g_{3}(x) .
\end{aligned}
$$

Here we have two kernels on the right and one on the left.

The conditions for the existence of a chain transform are of two kinds, (i) convergence conditions for the functions $g_{i}(x)$ and the kernels and (ii) a condition which must be satisfied by the kernels alone. We shall not be concerned here with the conditions satisfied by the functions $g_{i}(x)$. The kernels satisfy the following conditions: if $\mathfrak{M} f(x)$ denotes the Mellin transform of $f(x)$, see (8), (9) $\$ 3$, then

$$
\prod_{q} \mathfrak{M} l_{q}(x)=\prod_{p} \mathfrak{M} r_{p}(x),
$$

if there are kernels on both sides of (1), (2). If there are no kernels on the left of (1), (2) then the product on the left hand side of (6) must be replaced by 1 (unity) and if there are no kernels on the right of (1), (2) then the right hand product of (6) must be replaced by 1 (unity). The values of $q$ and $p$ in (6) are the same as the values of $q$ and $p$ which occur in the kernels of (1) and (2).

In (3), (4), (5) we have $r_{1}(x)=e^{-1 / x} / x$ and $\mathfrak{M} r_{1}(x)=\Gamma(1-s)$; $r_{2}(x)=e^{-x}$ and $\mathfrak{M} r_{2}(x)=\Gamma(s) ; l_{1}(x)=1 /(1+x)$ and $\mathfrak{M} l_{1}(x)=\pi / \sin (s \pi)$. Evidently (6) is satisfied since $\pi / \sin (s \pi)=\Gamma(1-s) \Gamma(s)$.

In addition to (6) the kernels must satisfy some convergence conditions; e.g. as all the kernels are in $L(0, \infty)$ or all are in $L_{2}(0, \infty)$, Fox [2, pp. 680,684]. If (6) is satisfied and one of these convergence conditions also holds we shall say that the kernels are the kernels of a chain transform.

3. Fractional integration. Various definitions of fractional integration have been given by several authors, including Riemann, Weyl and others. We shall follow the work of Kober [4] who has made a very comprehensive study of this operation. Kober defines four forms of fractional integration, but we shall discuss only the first one in detail since it is easy to establish theorems analogous to our Theorem 1 for the other three forms. If $\eta$ is a complex parameter then Kober defines the operator $I_{\eta, \alpha}^{+} f(t)$ as follows:

$$
g_{\eta, \alpha}^{+}(x)=I_{\eta, \alpha}^{+} f(t)=\frac{x^{-\eta-\alpha}}{\Gamma(\alpha)} \int_{0}^{x}(x-t)^{\alpha-1} t^{\eta} f(t) d t .
$$


(A) Kober proves the following result, [4, p. 199]; If

(i) $1 \leqq p<\infty$,

(ii) $R(\eta)>-1 / p$,

(iii) $R(\alpha)>0, f(t) \in L_{p}(0, \infty)$ then $g_{\eta, \alpha}^{+}(x)$ exists almost everywhere in $(0, \infty)$ and is also in $L_{p}(0, \infty)$.

The Mellin transform of $f(x)$, denoted by $\mathfrak{M} f(x)$, is defined as follows: if $1 \leqq p \leqq 2$ and $f(x) \in L_{p}(0, \infty)$ then

$$
\begin{array}{lrl}
\mathfrak{M}_{f}(x)=\int_{0}^{\infty} f(x) x^{s-1} d x, & (p=1) \\
\mathfrak{M}_{f}(x)=\text { l.i.m. } \int_{1 / N}^{N} f(x) x^{s-1} d x, & (1<p \leqq 2)
\end{array}
$$

where $s=(1 / p)+i$ and the limit in the mean has index $p /(p-1)$ $=p_{1}$. It is well known that $\mathfrak{M} f(x) \in L_{p_{1}}(-\infty, \infty)$. For the case $p=p_{1}$ $=2$ see Titchmarsh $[5, \S 3.17]$ and for the general case $1 \leqq p<2$ the result is deducible from corresponding theorems in Fourier Transform theory.

(B) Kober $[4, \S 4]$ proves that if

(i) $1 \leqq p \leqq 2$,

(ii) $f(x) \in L_{p}(0, \infty)$ and

(iii) $R(\eta)>(1-p) / p$ then

$$
\mathfrak{M} g_{\eta, \alpha}^{+}(x)=\mathfrak{M} I_{\eta, \alpha}^{+} f(t)=\frac{\Gamma(\eta+1-s)}{\Gamma(\eta+\alpha+1-s)} \mathfrak{M} f(x) .
$$

\section{Fractional integration of kernels.}

Theorem 1. If (i) the real parts of all the numbers $\alpha_{p}, \alpha_{q}, \eta_{p}$ and $\eta_{q}$ in (11) below are positive, (ii) the functions $r_{p}(x)$ and $l_{q}(x)$ are all in $L_{m}(0, \infty), m \geqq 1$, (iii) $r_{p}(x)$ are the right hand kernels and $l_{q}(x)$ are the left kernels of a chain transform and (iv)

$$
\prod_{q} \frac{\Gamma\left(\eta_{q}+1-s\right)}{\Gamma\left(\eta_{q}+\alpha_{q}+1-s\right)}=\prod_{p} \frac{\Gamma\left(\eta_{p}+1-s\right)}{\Gamma\left(\eta_{p}+\alpha_{p}+1-s\right)}
$$

then (a) all the functions $I_{\eta_{p}, \alpha_{p}}^{+} r_{p}(t)$ and $I_{\eta_{q}, \alpha_{q} l_{q}}^{+}(t)$ are in $L_{m}(0, \infty)$ and (b) they also form the right hand and left hand kernels, respectively, of a chain transform. Here $p$ and $q$ between them run through the first $n$ positive integers as explained in $\$ 2$.

Proof. From (A), immediately following (7), it is evident that (a) follows from condition (ii). To prove (b) we must establish (6) for the Mellin transforms of the $I_{\eta, \alpha}^{+}$functions. From (10) we have 


$$
\begin{aligned}
\prod_{q} \mathfrak{M} I_{\eta_{,}, \alpha_{q}}^{+} l_{q}(t) & =\prod_{q} \frac{\Gamma\left(\eta_{q}+1-s\right)}{\Gamma\left(\eta_{q}+\alpha_{q}+1-s\right)} \prod_{q} \mathfrak{M} l_{q}(t) \\
& =\prod_{p} \frac{\Gamma\left(\eta_{p}+1-s\right)}{\Gamma\left(\eta_{p}+\alpha_{p}+1-s\right)} \prod_{p} \mathfrak{M} r_{p}(t)
\end{aligned}
$$

from (11) condition (ii) and (6). On applying (10) to the right hand side of (13) we have

$$
\prod_{q} \mathfrak{M} I_{\eta_{q}, \alpha_{q}}^{+} l_{q}(t)=\prod_{p} M I_{\eta_{p}, \alpha_{p}}^{+} r_{p}(t) .
$$

Statement (b) now follows from (6) and (14) so that the functions $r_{p}(x)$ and $l_{q}(x)$ remain the kernels of a chain transform after they have been acted upon by the operators of fractional integration $I_{\eta, \alpha}^{+}$. This completes the proof of the theorem.

We note, from (a), that when new chain kernels are formed by means of fractional integration the convergence conditions remain unchanged. In [3] I prove chain transform theorems where the convergence conditions correspond to the values $m=1$ and $m=2$ in condition (ii).

For illustration we shall apply the theorem to the three kernels of the third order chain transform of (3), (4) and (5). We have $r_{1}(x)$ $=e^{-1 / x} / x, r_{2}(x)=e^{-x}, l_{1}(x)=1 /(1+x)$, all of which are in $L_{2}(0, \infty)$. The conditions of Theorem 1 are satisfied if $r_{1}(x)$ is unaltered and $r_{2}(x)$ and $l_{1}(x)$ are replaced by $I_{\eta, \alpha}^{+} r_{2}(t)$ and $I_{\eta, \alpha}^{+} l_{1}(t)$ respectively, where $\eta$ and $\alpha$ are the same for both functions. From (10) we have

$$
\begin{aligned}
\mathfrak{M} I_{\eta, \alpha}^{+} r_{2}(t) & =\frac{\Gamma(\eta+1-s)}{\Gamma(\eta+\alpha+1-s)} \Gamma(s), \\
\mathfrak{M} I_{\eta, \alpha}^{+} l_{1}(t) & =\frac{\Gamma(\eta+1-s)}{\Gamma(\eta+\alpha+1-s)} \frac{\pi}{\sin (s \pi)} .
\end{aligned}
$$

On applying the inverse Mellin transform to (15) and (16) we then obtain new kernels of a third order chain transform. Denoting these by $R_{1}(x), R_{2}(x)$ on the right hand side and $L_{1}(x)$ on the left we have

$$
\begin{aligned}
& R_{1}(x)=e^{-1 / x} / x, \\
& R_{2}(x)={ }_{1} F_{1}(\eta+1 ; \eta+\alpha+1 ;-x), \\
& L_{1}(x)={ }_{2} F_{1}(\eta+1,1 ; \eta+\alpha+1 ;-x) .
\end{aligned}
$$

Here the hypergeometric function ${ }_{2} F_{1}$ is given by the standard hypergeometric series when $0 \leqq x \leqq 1$ and by the analytic continuation of 
this series when $x>1$. The function ${ }_{1} F_{1}$ is a hypergeometric function of the Kummer type, Whittaker and Watson [6, Chapter XVI].

The chain transform (3), (4), (5) is related to the Stieltjes transform, Widder [7, Chapter 8]. Since the kernels (17), (18) and (19) have convergence properties which closely resemble those of (3), (4) and (5) it is probable that many of the results obtained in Stieltjes transform theory may also be true for chain transforms with kernels (17), (18) and (19).

5. Other forms of fractional integration. Kober [4] investigates three forms of fractional integration in addition to the one we have used here in $\S 3$. The second of these forms is as follows:

$$
\overline{h_{\eta, \alpha}}(x)=K_{\eta, \alpha}^{-} f(t)=\frac{x^{\eta}}{\Gamma(\alpha)} \int_{x}^{\infty}(t-x)^{\alpha-1} t^{-\eta-\alpha} f(t) d t
$$

with Mellin transform

$$
\mathfrak{M} h_{\eta, \alpha}^{-}(x)=\mathfrak{M} K_{\eta, \alpha}^{-} f(t)=\frac{\Gamma(\eta+s)}{\Gamma(\eta+\alpha+s)} \mathfrak{M} f(x) .
$$

The conditions for the existence of these functions differ slightly from those given in $A$ and $B \S 3$ above and for the case $p=2$, to which we confine ourselves, the conditions are exactly the same. The form (20) is associated with the Weyl method of fractional integration.

Evidently a theorem analogous to Theorem 1 can be established for the form of fractional integration given by (20). The result is stated here without proof.

Theorem 1A. If the conditions (i), (ii), (iii) and (iv) of Theorem 1 are true then the functions $K_{\eta_{p}, \alpha_{p}}^{-} r_{p}(t)$ and $K_{\eta_{q}, \alpha_{q}}^{-} l_{q}(t)$ are all in $L_{m}(0, \infty)$ and are, respectively, the right and left hand kernels of a chain transform of order $n$.

As explained in $\$ 2$ the $p$ 's and $q$ 's between them run through the first $n$ positive integers. For the purposes of proving this theorem it is more convenient to write condition (iii) of Theorem 1 with $1-s$ replaced by $s$.

Theorems analogous to Theorems 1 and $1 \mathrm{~A}$ can also be established for the other two forms of fractional integration discussed by Kober in $[4]$.

\section{REFERENCES}

1. A. Erdelyi, On fractional integration and its application to the theory of Hankel transforms, Quart. J. Math., Oxford Ser. (2) vol. 11 (1940) pp. 293-303.

2. C. Fox, Chain transforms, J. London Math. Soc. vol. 23 (1948) pp. 229-235. 
3. - Chain transforms, Proc. Amer. Math. Soc. vol. 5 (1954) pp. 677-688.

4. H. Kober, On fractional integrals and derivatives, Quart. J. Math. Oxford Ser. (2) vol. 11 (1940) pp. 193-211.

5. E. C. Titchmarsh, Introduction to the theory of Fourier integrals, Oxford, 1937.

6. E. T. Whittaker and G. N. Watson, Modern analysis, Cambridge, 1915.

7. D. Widder, The Laplace transform, Princeton, 1946.

McGill University

\section{SUR LES FONCTIONS DERIVEES, INTÉGRABLES AU SENS DE RIEMANN ET SUR LES DERIVEES PARTIELLES MIXTES}

\section{S. MARCUS}

1. Introduction. D'après A. P. Morse et W. W. Bledsoe, une fonction $f: S \rightarrow T$ (où $S$ et $T$ sont deux espaces métriques avec les distances $d$ et $d^{\prime}$ ) est dite envoisinée (neighborly) au point $x \in S$, si pour chaque $\epsilon>0$ il existe une sphère non vide et ouverte $U \subset S$, telle que pour tout $y \in U$ on ait $d(x, y)+d^{\prime}(f(x), f(y))<\epsilon,[1]$. Puisque $U$ n'est pas soumise à la condition de contenir le point $x$, la propriété ci-dessu est une généralisation de la notion de continuité. A. V. Martin énonce dans [7] (sans démonstration) le théorème suivant: "Si $f$ est une fonction réelle d'une variable réelle et si la dérivée $f^{\prime}$ existe et est intégrable au sens de Riemann sur tout intervalle compact, alors $f^{\prime}$ est envoisinée en chaque point." A. V. Martin affirme en [7] que ce théorème pourrait donner certaines suggestions concernant le problème, encore non résolu, de trouver une caractérisation intrinsèque des fonctions dérivées.

Dans la note présente, en utilisant l'équivalence de la notion de "fonction envoisinée" avec une notion introduite en 1933 par S. Kempisty (Théorème 1), on établit un théorème qui contient comme un cas particulier le résultat de A. V. Martin (Théorème 2). Puis, par certains exemples, on montre que la propriété d'être envoisinée est loin d'appartenir à chaque fonction dérivée, même bornée (Théorème 4), mais elle peut appartenir à une dérivée bornée qui n'est pas intégrable au sens de Riemann (Théorème 3 ). Etant donnée la liaison étroite entre les fonctions dérivées et les fonctions approximativement continues, on établit, pour ces dernières, un cas où elles sont envoisinées (Théorème 5). A la fin on donnent quelques applications au problème de la commutativité des dérivées partielles mixtes (Théorèmes 6 et 7).

Received by the editors March 26, 1958. 